Relations industrielles

Industrial Relations

\title{
Le salariat agricole au Québec
}

\section{Paul John Moran et Gilles Trudeau}

Volume 46, numéro 1, 1991

URI : https://id.erudit.org/iderudit/050649ar

DOI : https://doi.org/10.7202/050649ar

Aller au sommaire du numéro

Éditeur(s)

Département des relations industrielles de l'Université Laval

ISSN

0034-379X (imprimé)

1703-8138 (numérique)

Découvrir la revue

Citer cet article

Moran, P. J. \& Trudeau, G. (1991). Le salariat agricole au Québec. Relations industrielles / Industrial Relations, 46(1), 159-184.

https://doi.org/10.7202/050649ar
Résumé de l'article

Cet article s'intéresse aux salariés non familiaux du secteur agraire québécois. Il décrit d'abord les principales caractéristiques socio-économiques des ouvriers agricoles. Ensuite, il analyse dans quelle mesure le droit du travail québécois s'applique à ces travailleurs. Enfin, il discute de la validité des diverses exceptions et exclusions légales visant les salariés agricoles à la lumière des chartes canadienne et québécoise des droits de la personne.
Tous droits réservés @ C Département des relations industrielles de l'Université Laval, 1991
Ce document est protégé par la loi sur le droit d'auteur. L’utilisation des services d'Érudit (y compris la reproduction) est assujettie à sa politique d'utilisation que vous pouvez consulter en ligne.

https://apropos.erudit.org/fr/usagers/politique-dutilisation/ 


\title{
Le salariat agricole au Québec
}

\author{
Paul John Moran
}

et

\section{Gilles Trudeau}

Cet article s'intéresse aux salariés non familiaux du secteur agraire québécois. Il décrit d'abord les principales caractéristiques socio-économiques des ouvriers agricoles. Ensuite, il analyse dans quelle mesure le droit du travail québécois s'applique à ces travailleurs. Enfin, il discute de la validité des diverses exceptions et exclusions légales visant les salariés agricoles à la lumière des chartes canadienne et québécoise des droits de la personne.

Depuis quelques années, on assiste dans le domaine des relations du travail, à l'émergence, à l'organisation et à la manifestation de certains groupes de travailleurs*** offrant des caractéristiques particulières. Nous tenons notamment pour exemple les fonctionnaires, les policiers et les cadres. Traditionnellement, notre droit du travail avait répondu à ces caractéristiques en établissant des îlots protégés ou des régimes particuliers: exclusion pure et simple du régime général ou encore, exceptions par rapport aux règles générales. Or, les travailleurs concernés contestent de plus en plus les exceptions dont ils sont l'objet.

Ainsi, il y a déjà plus de vingt-cinq ans, les salariés de l'État québécois ont revendiqué et obtenu le droit à la syndicalisation et à la négociation collective. Plus récemment, certains groupes de policiers, principalement les membres de la Sûreté du Québec, ont contesté les particularités du régime

- MoRan, P.J., consultant, Les expertises ERG-S.S.T., Montréal. Montréal.

Trudeau, G. professeur agrégé, École des relations industrielles, Université de

** Les auteurs remercient monsieur Claude D'Aoust et madame Guylaine Vallée, professeurs à l'École de relations industrielles de l'Université de Montréal, pour leurs judicieux commentaires.

*** Dans ce document, la forme masculine désigne, lorsqu'il y a lieu, aussi bien les femmes que les hommes. L'emploi du masculin a pour unique but de faciliter la lecture du texte. 
auquel ils sont assujettis. Dans ce dernier cas, la Charte canadienne des droits et libertés, qui reconnaît le droit à l'égalité de chacun devant et dans la loi, constitue le fer de lance de la bataille judiciaire entreprise. On peut croire que d'autres travailleurs atypiques - les chauffeurs de taxi, par exemple - revendiqueront le droit aux bénéfices de notre législation en matière de travail.

Il est un groupe de salariés atypiques dont on entend peu ou jamais parler au Québec: les travailleurs ou les ouvriers agricoles. Pourtant, ils sont à l'oeuvre depuis longtemps chez nous. De plus, nos lois contiennent des dispositions spécifiques les excluant, totalement ou partiellement, de leur champ d'application.

Malgré l'abondance des études ayant abordé divers aspects de l'agriculture, bien peu d'auteurs se sont intéressés au phénomène du salariat agricole au Québec ${ }^{1}$. Cet article contribuera à combler ce vide dans la littérature en relations industrielles.

Dans un premier temps, nous nous proposons de soulever le voile qui recouvre la réalité du salariat agricole au Québec. Ainsi, nous tenterons de circonscrire l'ampleur du phénomène et de décrire sommairement les caractéristiques socio-économiques des travailleurs agricoles.

Ensuite, nous établirons dans quelle mesure les règles issues du droit du travail s'appliquent aux ouvriers agricoles québécois. Pour ce faire, nous passerons en revue tant le contenu de notre droit commun que celui propre au travail afin de dégager les règles qui visent exclusivement ou particulièrement ces travailleurs.

Enfin, nous discuterons brièvement de la validité des exclusions que contient notre législation du travail à l'égard de ces salariés à la lumière des chartes canadienne et québécoise des droits de la personne. Plus particulièrement, nous nous demanderons si ces exclusions respectent le droit à l'égalité de tous dans la loi, droit que ces chartes formulent.

\section{CARACTÉRISTIQUES SOCIO-ÉCONOMIQUES ET CONDITIONS DE TRAVAIL}

Cette première section traite du phénomène du salariat agricole et de ses principales caractéristiques actuelles. Nous y aborderons successivement le contexte et les caractéristiques sociologiques du salariat agricole et enfin, les conditions d'emploi des travailleurs agricoles au Québec. 
Le secteur agricole québécois a connu depuis la Seconde Guerre mondiale des modifications organisationnelles et commerciales fort importantes ${ }^{2}$. Aujourd'hui dotées de capitaux fonciers ou immobiliers relativement élevés et d'un haut degré de machinisme, les entreprises agricoles sont aussi devenues graduellement dépendantes d'un apport en main-d'oeuvre salariée. Dans un contexte d'expansion de la taille des fermes et d'un faible bilan démographique, les familles agricoles québécoises doivent donc de plus en plus recourir à des salariés externes à l'unité familiale.

Selon des calculs estimatifs, ces travailleurs ou ouvriers agricoles totalisent au minimum 15000 individus au Québec ${ }^{3}$ et ils forment, dans la perspective des relations industrielles, ce que nous entendons par le salariat agricole au Québec. Ces individus représentent approximativement $50 \mathrm{p}$. cent de la main-d'oeuvre agricole salariée québécoise; les membres rémunérés des familles agricoles et les contremaîtres, les gérants ou directeurs de ferme composent l'autre moitié des salariés agricoles au Québec.

Selon toute vraisemblance, les ouvriers agricoles se distribuent à peu près également entre travailleurs permanents et travailleurs saisonniers. Les premiers se retrouvent surtout dans les fermes centrées sur les productions animales alors que les seconds oeuvrent plutôt dans les établissements consacrés aux productions végétales.

Quant à la répartition des travailleurs agricoles par sexe et par groupe d'âge, il semble, grosso modo, que le salariat agricole est encore en majorité composé de jeunes adultes de sexe masculin ${ }^{4}$. Cependant, au cours des récentes années, la présence féminine tend à s'accroître, particulièrement dans les fermes spécialisées en horticulture. On peut également noter la présence d'adolescents dans certaines fermes maraîchères ou fruitières ${ }^{5}$.

Malgré l'impression généralement véhiculée dans les médias, le salariat agricole demeure très largement composé de Québécois de souche canadienne-française, ce qui n'empêche pas que plusieurs entreprises agricoles comptent également sur un nombre non négligeable de néo-Québécois, surtout les fermes horticoles de la grande région de Montréal ${ }^{6}$. Dans le cadre d'une pénurie grandissante d'ouvriers agricoles au Québec, on retrouve aussi dans ce même type de ferme un nombre croissant de travailleurs étrangers, particulièrement de nationalité mexicaine ${ }^{7}$.

D'autres calculs permettent également d'établir qu'environ 4150 fermes emploient des travailleurs agricoles permanents, soit une moyenne de 1,8 ouvriers à l'année par ferme déclarant une telle main-d'oeuvre. Par ailleurs, il n'y aurait tout au plus que $\mathbf{2 7 5}$ fermes employant trois travailleurs agricoles permanents ou plus, soit le nombre minimum et le type de salariés requis pour avoir accès à la syndicalisation dans le secteur agricole ${ }^{8}$. 
Du côté des quelque 14275 entreprises agricoles qui embauchent des travailleurs saisonniers', l'effectif par ferme varierait entre 1 et 100 ou même 150 salariés $^{10}$.

Le taux de syndicalisation dans cette industrie demeure extrêmement bas puisqu'au maximum 200 travailleurs agricoles, répartis dans environ une dizaine de fermes, seraient présentement syndiqués au Québec. Ce taux varie entre $0,75 \mathrm{p}$. cent et $3,75 \mathrm{p}$. cent, selon que l'on considère ou non les travailleurs saisonniers ${ }^{11}$. C'est donc dire qu'une très faible proportion du salariat agricole québécois est couverte par une convention collective.

En conséquence, la quasi-totalité des ouvriers ou travailleurs agricoles au Québec se fient aux lois du marché et à leur pouvoir individuel de négociation pour tenter d'établir leurs principales conditions d'emploi, dont le salaire et la durée de travail.

Selon certaines compilations effectuées par des responsables des Services de l'emploi agricole - des centres de main-d'oeuvre administrés par l'Union des producteurs agricoles (UPA), les ouvriers agricoles permanents ou à l'année gagnent entre $250 \$$ et $350 \$$ pour 60 heures en moyenne de travail par semaine ${ }^{12}$. De leur côté, les travailleurs saisonniers touchent à peu près le salaire horaire minimum pour une semaine de travail d'au moins 50 heures ${ }^{13}$. Leur période d'emploi varierait entre quelques semaines et six ou sept mois, au maximum.

L'ensemble des travailleurs agricoles n'auraient pas, sauf exception, de vacances payées ni de jours fériés. Tout au plus, la plupart des ouvriers agricoles permanents bénéficient de certains aliments gratuits et d'un logement à frais réduits ${ }^{14}$.

Ainsi, en dépit d'une pénurie chronique et générale d'ouvriers agricoles au Québec, ceux-ci gagnent des salaires côtoyant le salaire horaire minimum et ce, pour des durées de travail quotidienne et hebdomadaire qui excèdent sensiblement celles prévalant dans les autres secteurs économiques.

Au surplus, l'activité en milieu agricole présente des risques élevés en matière de santé et de sécurité au travail. En effet, au cours de la dernière décennie, le secteur agricole a connu en moyenne 14 décès accidentels par année ${ }^{15}$, plaçant ainsi globalement l'agriculture au troisième rang en ce qui concerne le taux des décès reliés au travail, après les industries des mines et de la construction. De plus, le taux d'incapacité permanente dans le secteur agricole est à peu près deux fois plus élevé que celui de l'ensemble des industries cotisant à la Commission de la santé et de la sécurité du travail ${ }^{16}$.

Ainsi, malgré l'image bucolique qui lui est souvent attribuée, l'agriculture apparaît une industrie où l'on retrouve de multiples et sérieuses 
menaces à la santé ou à l'intégrité physique des travailleurs, surtout en raison des équipements et des objets de travail qui sont propres au domaine agricole.

En somme, les ouvriers agricoles québécois sont relativement nombreux mais rarement syndiqués, et ils oeuvrent dans un secteur économique peu rémunérateur et à haut risque professionnel.

Ayant complété ce survol des principales caractéristiques socio-économiques et des conditions de travail des ouvriers agricoles au Québec, nous examinerons maintenant leur situation juridique en matière de travail. C'est l'objet de la prochaine section.

\section{L'ENCADREMENT JURIDIQUE DU TRAVAIL SALARIÉ AGRICOLE}

Dans quelle mesure les règles juridiques qui encadrent le travail salarié agricole diffèrent-elles des règles générales du droit du travail québécois? Cette section propose des éléments de réponse à cette question.

Pour ce faire, nous vérifierons si les principales constituantes de notre droit du travail s'appliquent au travail salarié agricole. Dans cette perspective, nous considérerons d'abord le contrat individuel de travail. Par la suite, nous analyserons les lois du travail d'application générale et, finalement, les règles des rapports collectifs du travail.

\section{Le contrat individuel de travail}

Les dispositions du Code civil se rapportant au contrat de louage de service personnel ${ }^{17}$ s'appliquent en général au travail salarié agricole. Malgré la formulation de certaines exceptions, elles ne créent pas un véritable régime particulier à l'égard des travailleurs de ce secteur.

Le texte même du Code civil reconnaît l'existence du travail salarié agricole. Par exemple, l'article 1669 permet à l'employeur, en l'absence de preuve écrite, d'offrir son serment «quant aux conditions de l'engagement et sur le fait du paiement, en l'accompagnant d'un état détaillé» comme preuve dans «toute action pour salaire par les domestiques ou serviteurs de ferme $\rangle^{18}$. Le troisième paragraphe de l'article 2262 fixe exceptionnellement le délai de prescription pour la réclamation des «gages des domestiques de maison ou de ferme» à une année ${ }^{19}$. Nous croyons que les expressions «serviteurs de ferme» et "domestiques [...] de ferme» visent des salariés que nous appelons aujourd'hui «travailleurs ou ouvriers agricoles». 
Le contrat individuel de travail demeure la source la plus importante des règles juridiques s'appliquant au travail de bon nombre d'ouvriers agricoles au Québec. En effet, comme le démontre la section suivante, plusieurs lois générales du travail ne s'appliquent pas indistinctement à tous les salariés du secteur agricole.

\section{Les lois du travail d'application générale}

Dans quelle mesure les principales lois québécoises en matière de travail s'appliquent-elles aux ouvriers agricoles? C'est l'objet de cette sous-section.

\section{La Loi sur les normes du travail}

La Loi sur les normes du travail ${ }^{20}$ établit des conditions minimales de travail en deça desquelles il est illégal de faire effectuer un travail salarié. Le champ d'application de la loi est très large ${ }^{21}$ et ses dispositions sont d'ordre public $^{22}$. Bien que d'application très générale, la Loi sur les normes $d u$ travail formule certaines exceptions à cet égard. Son article troisième, paragraphe premier, mentionne qu'elle ne s'applique pas

au salarié employé à l'exploitation d'une ferme mise en valeur:

a) par une personne physique seule ou avec son conjoint ou un descendant ou un ascendant de l'un ou l'autre, avec le concours habituel d'au plus trois salariés;

b) par une corporation dont c'est l'activité principale avec le concours habituel d'au plus trois salariés en sus des trois principaux actionnaires de la corporation s'ils y travaillent;

c) par une société ou par des personnes physiques agissant en copropriété, avec le concours habituel d'au plus trois salariés.

Telle que rédigée, cette disposition écarte du champ d'application de la loi la grande majorité des fermes et leurs salariés. L'ancienne Loi du salaire minimum $^{23}$, en vigueur jusqu'en avril 1980, excluait, quant à elle, les «salariés travaillant à des exploitations agricoles», ces dernières signifiant "une ferme mise en valeur par l'exploitant lui-même, ou par l'entremise d'employés» ${ }^{24}$. Malgré son libellé plus long, l'exclusion apparaissant dans la Loi sur les normes du travail n'est pas très différente de celle contenue dans la loi antérieure, ce que souhaitait d'ailleurs l'Union des producteurs agricoles, l'organisme légalement représentatif de tous les producteurs et employeurs agricoles du Québec ${ }^{25}$.

Quoique superficiellement, une seule décision judiciaire interprète l'expression «exploitation d'une ferme» de l'article 3 de la Loi sur les normes 
du travail. Celle-ci laisse entrevoir que cette expression traduit surtout l'idée du travail du sol ou de sa culture et ce, en vue de la production d'un bien agricole $^{26}$. Dans ces circonstances, la jurisprudence développée sous la loi précédente devrait prévaloir et imposer une notion large de l'exploitation agricole. En effet, le législateur vise l'exploitation de la ferme et non seulement les travaux courants de la ferme. Ceci devrait permettre d'inclure certaines activités commerciales directement reliées à la ferme ${ }^{27}$. L'entreprise d'élevage d'animaux de ferme est sans aucun doute aussi incluse dans la notion d' «exploitation d'une ferme» ${ }^{28}$.

Cette notion s'entend également d'une mise en valeur «avec le concours habituel d'au plus trois salariés». Pour conclure à l'assujettissement d'une «exploitation d'une ferme» à la Loi sur les normes de travail, il faut donc démontrer qu'elle fonctionne avec l'aide constante et régulière d'au moins quatre salariés ${ }^{29}$. On ne doit pas tenir compte des salariés occasionnels embauchés régulièrement, année après année, pour une période de temps limitée ${ }^{30}$.

Enfin, dans le cas d'une entreprise constituée en corporation, l'exclusion générale ne s'applique que si l'exploitation agricole en représente l'activité principale. Dans le cas contraire, l'entreprise incorporée demeure assujettie à la loi même si elle embauche moins de quatre salariés ${ }^{31}$.

Par ailleurs, au delà de l'exclusion générale formulée à l'article 3 de la loi, la Loi sur les normes du travail et la réglementation ont créé d'autres mesures d'exception concernant le salarié d'une exploitation agricole. Ainsi, le «travailleur agricole»" ${ }^{32}$ et le "salarié affecté à la récolte, à la mise en conserve, à l'empaquetage et à la congélation des fruits et des légumes, pendant la période des récoltes» ${ }^{33}$ ne sont pas protégés par la loi en matière de semaine normale de travail ou de paiement d'heures supplémentaires.

Il en est ainsi pour tout "salarié surnuméraire pendant la période des récoltes» en ce qui concerne le congé annuel ou la prime de vacances ${ }^{34}$. Quant au repos hebdomadaire minimal de 24 heures consécutives, le deuxième alinéa de l'article 78 de la loi mentionne que seulement «[d]ans le cas d'un travailleur agricole, ce jour de repos peut être reporté à la semaine suivante.» Ainsi, tout ouvrier agricole peut, en toute légalité, être contraint de travailler douze jours consécutifs, même s'il est, par ailleurs, couvert par la Loi sur les normes du travail. C'est là, à notre avis, une exception qui ne peut pas se justifier eu égard à la santé et à la sécurité au travail.

Le Règlement sur les normes du travail ${ }^{35}$ établit que le salaire minimum ne s'applique pas au «salarié surnuméraire embauché sur une base occasionnelle pour les récoltes», ni au «salarié employé aux productions fruitières ou horticoles et affecté principalement à des opérations non mécanisées» ${ }^{36}$. De 
plus, la loi réserve au gouvernement la possibilité de soustraire par règlement tout travailleur agricole à l'application des normes portant sur le salaire minimum, les modalités de paiement du salaire, le bulletin de paye, les retenues autorisées sur le salaire et les frais d'hébergement ${ }^{37}$.

Cette brève analyse nous permet de conclure qu'à toutes fins utiles, l'ouvrier agricole est exclu de la protection de la Loi sur les normes du travail. Il y a d'abord l'exclusion générale des fermes comptant moins de quatre salariés engagés à l'année. Cette exclusion affecte la grande majorité des ouvriers agricoles, en raison du faible nombre moyen de travailleurs agricoles permanents par ferme ${ }^{38}$. Ensuite, il y a plusieurs exclusions spécifiques contenues dans la loi ou la réglementation qui écartent de nombreux salariés agricoles, ou certaines catégories d'entre eux, de l'application de normes du travail fort importantes ${ }^{39}$.

En terminant cette courte étude de la Loi sur les normes du travail, mentionnons que la Loi sur la fête nationale ${ }^{40}$ qui consacre le 24 juin, la Saint-Jean-Baptiste, comme fête nationale des Québécois et qui en fait un jour chômé et payé, s'applique aussi aux travailleurs agricoles, sous réserve de l'application de la loi elle-même ${ }^{41}$. En effet, aucune exclusion spécifique ne les concerne.

\section{La Loi sur la santé et la sécurité du travail}

La Loi sur la santé et la sécurité du travail ${ }^{42}$ définit largement son champ d'application. À cet égard, aucune exclusion ou exception ne vise les travailleurs agricoles et leurs employeurs. Les ouvriers de ce secteur bénéficient donc des avantages généraux de cette importante loi, comme l'ensemble des autres salariés québécois. Les droits et obligations que cette loi définit à l'égard des travailleurs et des employeurs apparaissent primordiaux lorsque considérés dans le cadre de l'agriculture, une industrie particulièrement dangereuse $\mathrm{e}^{43}$.

Notons cependant que l'industrie agricole n'est pas un secteur désigné par le règlement relatif au programme de prévention, ni celui prévoyant la formation d'un comité de santé et de sécurité, non plus que celui régissant la désignation d'un représentant à la prévention ${ }^{44}$.

\section{La Loi sur les accidents du travail et les maladies professionnelles}

Cette loi établit le régime québécois d'indemnisation des victimes d'une lésion professionnelle ${ }^{45}$. Elle a été précédée par la Loi des accidents du travail, sanctionnée en $1931^{46}$, qui formulait les grands principes d'indemnisation et de responsabilité que l'on retrouve encore aujourd'hui. Cependant, le secteur agricole était spécifiquement exclu du régime établi par la loi de 
1931. Ce n'est qu'en 1978 qu'on abrogea cette exclusion ${ }^{47}$. Sous l'insistance et avec le concours de l'Union des producteurs agricoles, la loi s'appliqua

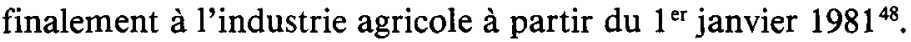

La Loi sur les accidents du travail et les maladies professionnelles ne comporte donc pas d'exclusion ou d'exception visant l'industrie agricole. Les travailleurs et les employeurs de ce secteur participent ainsi obligatoirement au régime qu'elle établit.

Ceci termine notre brève revue des règles de droit commun et de celles découlant des principales lois d'application générale à l'égard du travail salarié agricole. Regardons maintenant dans quelle mesure les lois qui traitent des rapports collectifs du travail au Québec s'appliquent aux travailleurs agricoles et à leurs employeurs.

\section{Les rapports collectifs du travail}

Deux lois doivent être considérées sous cette rubrique: le Code $d u$ travail et la Loi sur les décrets de convention collective.

\section{Le Code du travail}

Les travailleurs agricoles ne font pas l'objet d'une exclusion générale dans le Code du travail $^{49}$ : ils sont implicitement compris dans la définition de «salarié» de l'article $1(1)^{50}$. Cependant, le cinquième alinéa de l'article 21 se lit comme suit:

Les personnes employées à l'exploitation d'une ferme ne sont pas réputées être des salariés aux fins de la présente section, à moins qu'elle n'y soient ordinairement et continuellement employées au nombre minimal de trois ${ }^{51}$.

La grande majorité des ouvriers agricoles ne peut donc former une association de salariés dans le but d'obtenir l'accréditation et ainsi obliger l'employeur à négocier de bonne foi leurs conditions de travail. En quelque sorte, ces travailleurs sont privés des principaux avantages du Code $d u$ travail.

On retrouve dans la jurisprudence quelques décisions interprétant cette disposition. Ainsi, en appel d'une décision d'un commissaire-enquêteur accordant une accréditation, le Tribunal du travail jugea qu'une coopérative de producteurs agricoles n'était pas «une ferme en exploitation» mais bien «une entreprise d'affaires qui a une main-d'oeuvre totalement salariée» et en conséquence, il confirmait le jugement rendu antérieurement ${ }^{52}$. 
En 1980, ce même tribunal décida qu' «une entreprise commerciale même si celle-ci comporte partiellement une activité agricole» n'était pas équivalente à «l'exploitation d'une ferme»: même si ses salariés d'entrepôt oeuvraient occasionnellement dans les fermes qu'exploitait cette entreprise, la requête en accréditation de leur syndicat était recevable, tel que d'abord considéré par un commissaire-enquêteur ${ }^{53}$.

Plus récemment, le Tribunal du travail confirmait l'accréditation d'une association de salariés comportant entre autres «deux fermiers qui [recevaient] une rémunération sous forme de salaire» et ce, parce que leur employeur n'était pas «une entreprise d'exploitation de ferme mais une entreprise d'enseignement [...] [qui] accessoirement [...] possèd[ait] une ferme $)^{54}$. Le juge Morin ajoutait en obiter que:

La mention d'un nombre d'employés qui sert à déterminer l'existence ou non de l'exception [apparaissant à l'alinéa 5 de l'article 21 du Code du travail], démontre que le but visé par le législateur était d'exclure le droit d'accréditation que pour les petites entreprises [agricoles]. Lorsqu'on parle d'entreprises exploitant une ferme avec moins de trois employés, on réfère principalement aux entreprises familiales possédant une ferme. Ce sont principalement les fermes exploitées par les membres d'une même famille ou par des parents que l'on n'a pas voulu sujettes à une accréditation. [...] [Le législateur] a voulu relier l'exception non pas au travail, aux fonctions occupées, mais à l'exploitation d'une ferme, à l'entreprise ${ }^{55}$.

À notre avis, il ressort de ces quelques décisions que les entreprises connexes à l'agriculture, même celles ayant accessoirement une activité agricole, sont assujetties, le cas échéant, à toutes les dispositions du Code du travail et ce, peu importe le nombre de leurs employés. Cependant, on n'a toujours pas décidé du sens de l'article 21(5) lorsque moins de trois salariés sont embauchés par une entreprise agricole incorporée ou dirigée par une société formée sous l'égide du Code civil. En l'absence d'une définition du mot «ferme» dans le Code du travail, on peut croire que de telles entreprises agricoles seraient considérées comme «l'exploitation d'une ferme» au même titre que celle possédée par un seul individu ou par une "personne physique». Ces salariés seraient donc vraisemblablement exclus de l'application du Code.

Enfin, signalons qu'il faut qu'au moins trois personnes «soient ordinairement et continuellement employées» à l'exploitation d'une même ferme avant de songer à l'obtention d'une accréditation syndicale. Comme l'épithète «habituel» dans la Loi sur les normes du travail, l'expression "ordinairement et continuellement» dans le Code du travail révèle l'intention du législateur d'assujettir à la loi les seules entreprises agricoles qui emploient un nombre minimal de salariés de façon permanente ou à l'année. 
$\mathrm{Vu}$ le faible nombre moyen de travailleurs agricoles permanents par ferme au Québec, nous estimons que, tout comme dans le cas de la Loi sur les normes du travail, la portée du Code du travail est encore aujourd'hui fort réduite dans le secteur agricole québécois. Cela expliquerait en partie le faible taux de syndicalisation observé dans ce secteur ${ }^{56}$.

\section{La Loi sur les décrets de convention collective}

Cette dernière loi ${ }^{57}$ exclut de son champ d'application les «exploitations agricoles», lesquelles correspondent à «une ferme mise en valeur par l'exploitant lui-même ou par l'entremise d'employés"s ${ }^{58}$.

L'extension juridique des conventions collectives, surtout utilisée dans des secteurs où prédomine la petite entreprise ${ }^{59}$, est donc pour le moment inapplicable à l'industrie agricole québécoise. Cette industrie est pourtant largement composée d'établissements embauchant un nombre réduit de travailleurs.

Ce tour d'horizon du droit du travail québécois permet de conclure que les ouvriers agricoles bénéficient, sur un plan juridique, d'un régime de travail moins favorable que les autres travailleurs du Québec. Dans la prochaine section, nous discuterons de la validité de ce traitement différent à la lumière des chartes canadienne et québécoise des droits de la personne.

\section{LES EXCLUSIONS DES OUVRIERS AGRICOLES À LA LUMIÈRE DES CHARTES}

Le statut particulier que notre législation du travail impose aux ouvriers agricoles est-il valide eu égard à la Charte canadienne des droits et libertés et à la Charte des droits et libertés de la personne du Québec? Nous apporterons quelques éléments de réponse à cette question dans les pages qui suivent. Le grand nombre de règles juridiques en matière constitutionnelle et leur complexité nous limitent cependant à ne procéder qu'à un bref survol du droit applicable.

\section{La Charte canadienne des droits et libertés}

La Charte canadienne des droits et libertés ${ }^{60}$ fait partie de notre constitution depuis 1982. Elle établit certains droits fondamentaux qu'elle protège de toute limitation injustifiée imposée par le gouvernement ou le législateur, qu'il soit fédéral, provincial ou organe à compétence réglementaire. Ce sont les tribunaux ${ }^{61}$ qui doivent trancher tout litige où est invoquée une violation de la Charte. 
La vérification de la validité d'une loi ou d'une action gouvernementale par rapport à la Charte se fait normalement en deux étapes. Le tribunal doit d'abord décider si l'action contestée restreint un droit ou une liberté protégée par la Constitution. S'il en conclut ainsi et qu'il s'agit d'une loi ou d'un règlement, le tribunal doit ensuite vérifier si cette restriction se situe «dans des limites qui soient raisonnables et dont la justification puisse se démontrer dans le cadre d'une société libre et démocratique»" ${ }^{62}$.

À notre avis, la constitutionnalité des diverses exclusions visant les travailleurs agricoles contenues dans notre législation du travail peut être mise en doute sur la base de l'article 15 de la Charte ${ }^{63}$. L'alinéa premier de cette disposition se lit comme suit:

La loi ne fait acception de personne et s'applique également à tous, et tous ont droit à la même protection et au même bénéfice de la loi, indépendamment de toute discrimination, notamment des discriminations fondées sur la race, l'origine sociale ou ethnique, la couleur, la religion, le sexe, l'âge ou les déficiences physiques ou mentales.

À notre connaissance, aucune décision n'a encore été rendue au Canada sur la validité du traitement différent fait aux ouvriers agricoles par plusieurs lois canadiennes du travail. Cependant, un syndicat a contesté la constitutionnalité de l'exclusion complète des travailleurs agricoles du Labour Relations Act ${ }^{64}$ de l'Ontario en invoquant l'article 15 de la Charte devant le Labour Relations Board ontarien. Cet organisme n'as pas encore officiellement abordé cette question, le débat ayant jusqu'à maintenant porté exclusivement sur la compétence du Board à se prononcer sur la validité constitutionnelle de la loi qu'il administre ${ }^{65}$. Par contre, certains principes de la jurisprudence actuelle peuvent orienter notre réflexion sur la question.

La Cour suprême du Canada a récemment interprété l'article 15 de la Charte dans l'affaire The Law Society of British Columbia et al. c. Andrews et al. ${ }^{66}$. Dans cette décision, notre plus haute cour a établi plusieurs paramètres quant à la portée de cette importante disposition de la Charte.

Il appartient d'abord au citoyen qui conteste la constitutionnalité d'une loi ou d'une décision gouvernementale d'établir que le droit à l'égalité conféré par l'article 15(1) a été restreint. Or, ce droit à l'égalité n'est pas violé par toute distinction ou traitement différent consacré dans une loi: il faut aussi que la distinction soit discriminatoire. Le juge McIntyre, dans une opinion qui semble rallier sur ce point l'unanimité des juges ayant participé à la décision Andrews, définit la discrimination comme:

une distinction, intentionnelle ou non, mais fondée sur des motifs relatifs à des caractéristiques personnelles d'un individu ou d'un groupe d'individus, qui a pour 
effet d'imposer à cet individu ou à ce groupe des fardeaux, des obligations ou des désavantages non imposés à d'autres ou d'empêcher ou de restreindre l'accès aux possibilités, aux bénéfices et aux avantages offerts à d'autres membres de la sociétét ${ }^{67}$. (L'italique est de nous)

L'article 15(1) identifie certains motifs de discrimination qui sont illicites. Ce sont tous des motifs reliés aux caractéristiques personnelles d'un individu. Cette énumération n'est cependant pas exhaustive. Dans l'affaire Andrews, la Cour suprême refuse de préciser ou de limiter les autres facteurs qui pourraient être invoqués ${ }^{68}$. On ne sait toujours pas aujourd'hui si les autres facteurs, ceux non énumérés, doivent être analogues à ceux qui sont expressément nommés. Jusqu'à maintenant, la Cour suprême a seulement reconnu des facteurs analogues de discrimination même si la porte n'est pas complètement fermée aux autres motifs ${ }^{69}$.

Une fois la violation du droit à l'égalité démontrée, l'État peut tenter de justifier cette dérogation en utilisant l'article 1 de la Charte. C'est la décision qu'a rendue la Cour suprême du Canada dans l'affaire La Reine c. Oakes $^{70}$ qui précise le contenu de la preuve à apporter. Premièrement, l'État doit démontrer l'existence d'une politique ou d'un objectif suffisamment important pour justifier la suppression d'un droit ou d'une liberté garantis par la Constitution ${ }^{71}$. A ce sujet, le juge en chef Dickson écrit, au nom de la Cour:

[i]l faut à tout le moins qu'un objectif se rapporte à des préoccupations urgentes et réelles dans une société libre et démocratique, pour qu'on puisse le qualifier de suffisamment important ${ }^{72}$.

Une fois un tel objectif établi, il faut démontrer que les moyens choisis sont raisonnables et justifiables. Pour ce faire, on doit soupeser les intérêts de la société et ceux des individus ou des groupes visés. Ce test de proportionnalité, selon la Cour, comporte trois volets: 1) les mesures sont conçues pour atteindre l'objectif invoqué; 2) elles doivent porter le moins possible atteinte au droit ou à la liberté en question; 3) les effets des mesures choisies doivent être proportionnels à l'objectif poursuivi ${ }^{73}$.

Compte tenu de ce qui précède, 1'article 15 (1) de la Charte canadienne offre-t-il un recours utile pour contester les exclusions visant les ouvriers agricoles? Il n'y a aucun doute que ces travailleurs subissent un traitement différent de celui dont bénéficient les salariés des autres secteurs d'activités en vertu de notre législation du travail. L'analyse que nous avons présentée dans la deuxième partie de cet article le démontre clairement. Toutefois, ce traitement différent, cette discrimination professionnelle, constitue-t-il de la discrimination au sens de l'article 15(1)? 
La distinction dont sont l'objet les ouvriers agricoles les prive des bénéfices souvent nécessaires, voire essentiels, que la législation du travail accorde à d'autres travailleurs qui, face à la partie patronale, sont placés dans des situations similaires. Cette distinction est donc désavantageuse à l'égard des ouvriers agricoles. Cependant, elle n'est pas soutenue par des motifs qui sont énumérés explicitement à l'article 15(1). Elle n'est pas non plus fondẻe sur d'autres facteurs analogues, c'est-à-dire des motifs reliés aux caractéristiques personnelles de ces ouvriers. La distinction est plutôt établie à partir de l'activité économique que pratique l'employeur. C'est parce que ces travailleurs participent à titre de salariés à l'exploitation d'une ferme ou parce qu'ils font du travail agricole qu'ils sont l'objet des exclusions décrites plus haut.

Nous avons déjà mentionné que la Cour suprême a refusé de limiter les motifs qui peuvent engendrer de la discrimination au sens de l'article 15(1). Même si jusqu'à maintenant, la Cour suprême n'a reconnu que des motifs reliés aux caractéristiques personnelles, la possibilité demeure donc qu'elle perçoive comme discriminatoire un traitement différent imposé en raison du secteur d'activités dans lequel intervient un travailleur ou de la nature de son travail ${ }^{74}$.

$\mathrm{Si}$, à l'intérieur d'une instance, le tribunal détermine que le traitement différent fait aux ouvriers agricoles par une disposition légale restreint le droit à l'égalité énoncé à l'article 15(1), l'État pourra tenter de justifier cette restriction en invoquant l'article 1 de la Charte. La justification présentée devra respecter les conditions du test élaboré dans l'affaire Oakes. Ce test est fort exigeant et requiert de l'État qu'il démontre l'existence d'un objectif suffisamment important pour justifier le retrait de la protection de notre législation du travail aux ouvriers agricoles.

Dans le peu d'espace dont nous disposons, il est impossible de faire une analyse poussée de cette question. Mentionnons simplement que les exclusions visant les travailleurs agricoles résultent surtout d'un lobbying puissant des producteurs agricoles ou des propriétaires de ferme. Ces derniers sont, partout en Amérique du Nord, fort bien organisés et représentés. À l'opposé, les travailleurs ou les ouvriers agricoles comptent parmi les plus démunis et les plus isolés de notre société. Dans ces conditions, le lobbying patronal auprès des instances politiques a été jusqu'ici particulièrement efficace $^{75}$.

Les justifications qu'on pourrait avancer dans le cadre de l'article 1 sont surtout les suivantes: la protection du caractère particulier de la ferme familiale comme institution; l'augmentation des coûts de main-d'oeuvre qui 
résulterait de l'inclusion des travailleurs agricoles dans le régime légal général, hausse que plusieurs petites fermes ne pourraient ni supporter ni transmettre aux consommateurs; la grande flexibilité d'opération exigée par la nature saisonnière et la dépendance climatique de l'industrie agricole et enfin, la faible spécialisation de la main-d'oeuvre actuelle ${ }^{76}$.

Quoique toutes ces raisons soient sujettes à controverse, il serait surprenant, à la lumière de la décision Oakes, qu'elles puissent, même globalement, justifier les exclusions visant les ouvriers agricoles dans notre législation du travail.

\section{La Charte des droits et libertés de la personne du Québec}

Les dispositions de la Charte des droits et libertés de la personne du Québec ${ }^{77}$ permettent aussi d'envisager une contestation de la validité des exclusions dont sont victimes les ouvriers agricoles dans notre législation du travail. C'est en se fondant sur le droit à l'égalité énoncé à l'article $10 \mathrm{de}$ la charte, que cette contestation pourrait réussir ${ }^{78}$.

L'article 10 se lit comme suit:

Toute personne a droit à la reconnaissance et à l'exercice, en pleine égalité, des droits et libertés de la personne, sans distinction, exclusion ou préférence fondée sur la race, la couleur, le sexe, la grossesse, l'orientation sexuelle, l'état civil, l'âge sauf dans la mesure prévue par la loi, la religion, les convictions politiques, la langue, l'origine ethnique ou nationale, la condition sociale, le handicap ou l'utilisation d'un moyen pour pallier ce handicap.

Il y a discrimination lorsqu'une telle distinction, exclusion ou préférence a pour effet de détruire ou de compromettre ce droit. (L'italique est de nous)

Le droit à l'égalité de l'article 10 n'est pas une fin en soi: ce n'est pas un droit autonome. Il s'agit plutôt du droit à l'égalité dans la reconnaissance et l'exercice des droits et libertés de la personne. De plus, ce droit à l'égalité n'est violé que si la distinction ou le traitement différent contesté est fondé sur un motif illégal. Or, ces motifs sont énumérés limitativement à l'article $10^{79}$.

Concrètement, pour contester une disposition d'une loi sur la base de l'article 10, il faut établir trois éléments de preuve essentiels ${ }^{80}$.

En effet, il faut d'abord démontrer une «distinction, exclusion ou préférence», comme le mentionne l'article 10 . Nul doute que notre législation du travail renferme des distinctions et des exclusions à l'égard des ouvriers agricoles. 
En deuxième lieu, cette distinction ou exclusion doit être fondée sur un des motifs énumérés au premier alinéa de l'article 10. Dans le cas des travailleurs agricoles, on pourrait invoquer qu'on se base sur leur «condition sociale» pour les traiter de façon différente. Selon la Cour d'appel, la définition généralement acceptée de condition sociale

réfère soit au rang, à la place, à la position qu'occupe un individu dans la société, [...] de par sa naissance, de par son revenu, de par son niveau d'éducation, de par son occupation; soit à l'ensemble des circonstances et des événements qui font qu'une personne ou qu'un groupe occupe telle situation ou telle position dans la société $^{81}$. (L'italique est de nous)

Cette définition nous semble englober certaines formes de discrimination professionnelle. Il faut cependant admettre que, jusqu'à maintenant, les tribunaux ont interprété plutôt restrictivement la notion de condition sociale $^{82}$. Il n'est toutefois pas impossible qu'on accepte un jour d'inclure dans cette notion la position qu'une personne occupe dans la société en raison de son occupation professionnelle ${ }^{83}$.

Enfin, cette distinction doit compromettre le droit à l'égalité dans «la reconnaissance» et «l'exercice» d'un droit ou d'une liberté de la personne. Dans le cas des travailleurs agricoles, le droit fondamental implicitement énoncé dans l'article 16 de la Charte nous semble être compromis par le contenu particulier de la législation du travail à l'égard de ces salariés. Cette disposition se lit:

[n]ul ne peut exercer de discrimination dans l'embauche, l'apprentissage, la durée de la période de probation, la formation professionnelle, la promotion, la mutation, le déplacement, la mise à pied, la suspension, le renvoi, ou les conditions de travail d'une personne ainsi que dans l'établissement de catégories ou de classifications d'emploi. (L'italique est de nous)

En somme, on peut prétendre que notre législation du travail est discriminatoire et contraire à la Charte des droits et libertés de la personne lorsqu'elle édicte un régime et des conditions de travail distincts pour les ouvriers agricoles. Il en sera jugé ainsi dans la mesure où on démontrerait que cette distinction est effectivement fondée sur la condition sociale de ces travailleurs.

Cette revue sommaire du contenu des chartes canadienne et québécoise des droits de la personne et de la jurisprudence qui en découle nous permet de mettre sérieusement en doute la validité des dispositions de notre législation actuelle du travail qui excluent, totalement ou en partie, les travailleurs agricoles. Cette présumée violation est d'autant plus grave qu'elle est exercée par le législateur et que ces chartes reflètent des valeurs fondamentales de notre société. 


\section{CONCLUSION}

Nous cherchions, par cet article, à jeter un éclairage nouveau sur le phénomène du salariat agricole au Québec. Ainsi, nous pouvons constater que les ouvriers agricoles forment un groupe relativement nombreux: au moins 15000 personnes en $1986^{84}$. Les travailleurs agricoles permanents comme les ouvriers agricoles saisonniers sont rarement syndiqués, leur rémunération est peu élevée et ils sont confrontés à des risques professionnels importants.

Notre législation du travail, pourtant volumineuse et souvent avantgardiste, prive ces salariés agricoles de plusieurs des bénéfices qu'elle accorde aux autres travailleurs québécois. Par exemple, deux des lois les plus importantes en matière de travail, la Loi sur les normes du travail et le Code du travail, excluent de leur champ d'application les ouvriers travaillant dans des exploitations agricoles comptant moins de quatre salariés à l'année ou, dans le cas du Code, moins de trois employés permanents ${ }^{85}$. Ces exclusions visent la grande majorité des travailleurs agricoles puisqu'il n'y aurait, en moyenne, que 1,8 ouvriers à l'année par ferme déclarant une telle main-d'oeuvre au Québec ${ }^{86}$. Par ailleurs, une autre loi non négligeable en matière de travail, la Loi sur les décrets de convention collective, exclut sans distinction toutes les exploitations agricoles de son champ d'application.

Les travailleurs agricoles sont, par contre, assujettis à la Loi sur les accidents du travail et les maladies professionnelles. Même si leur admissibilité à ce régime est relativement récente, les salariés agricoles victimes d'une lésion professionnelle ont droit à une indemnisation déterminée à partir des mêmes règles que celles visant l'ensemble des travailleurs québécois. De la même façon, la Loi sur la santé et la sécurité du travail s'applique intégralement aux ouvriers agricoles. Toutefois, en dépit des hauts taux de risques professionnels qu'on y observe, le secteur agricole n'est toujours pas inclus dans les groupes prioritaires établis par cette loi.

En quelque sorte, ces différentes exclusions placent la vaste majorité des salariés agricoles dans une situation identique à celle que vivait l'ensemble des travailleurs au début de ce siècle: soumis aux aléas d'une liberté contractuelle quasi totale. En effet, les conditions de travail des ouvriers agricoles exclus de la législation du travail sont nécessairement et presqu'entièrement déterminées par le contenu de leur contrat individuel de travail. Force nous est de reconnaître que ce type de contrat, ou le droit commun qui le soutient, accorde peu de protection contre l'exploitation et l'abus à un travailleur dont les caractéristiques professionnelles ne lui confèrent pas un pouvoir de négociation significatif face à un employeur. 
Les dispositions particulières de notre législation du travail à l'égard des ouvriers agricoles ne sont pas à l'abri d'une contestation judiciaire. Le droit à l'égalité de tous devant et dans la loi, énoncé dans la Charte canadienne des droits et libertés ainsi que dans la Charte des droits et libertés de la personne du Québec, pourrait être invoqué devant les tribunaux. On peut sérieusement plaider que nos lois et règlements du travail limitent indûment ce droit fondamental eu égard aux travailleurs agricoles. Cependant, le résultat d'un tel débat judiciaire demeure incertain, compte tenu des conditions auxquelles la loi et la jurisprudence ont soumis le droit à l'égalité.

Nous croyons le temps venu de remettre en question de façon plus large les exclusions et exceptions légales dont sont l'objet les ouvriers agricoles. Ce débat devrait sous peu être refait - sinon aussitôt entrepris - au sein même des instances législatives appropriées. Les conditions sociales et économiques qui prévalaient au moment de leur adoption ne sont certainement plus les mêmes aujourd'hui. Sans pour autant rejeter péremptoirement les motifs qui pourraient être invoqués pour qu'elles soient maintenues, ces exclusions ou exceptions à l'endroit de travailleurs parmi les plus démunis de notre société nous apparaissent contestables, voire humiliantes.

\section{NOTES}

1 Notons cependant que ce phénomène fait depuis peu l'objet de quelques recherches universitaires: Guy DUCHARME, Le développement du salariat dans le secteur laitier québécois, thèse de M.Sc. (économie rurale), Université Laval, 1989, 260 p. (au moment d'écrire ces lignes (juillet 1990), ce mémoire n'a pas encore reçu d'approbation officielle et il n'était donc pas disponible pour consultation); Paul John MoRAN, Contexte industriel et caractéristiques générales et juridiques du salariat agricole au Québec, thèse de M.Sc. (relations industrielles), Université de Montréal, 1988, 329 p.; Michel MorISSET, Agriculture familiale ou capitaliste au Québec au $X X^{i e ̀ m e}$ siècle?, thèse de doctorat d'État (sciences économiques), Université de Paris VIII (Vincennes), 1982, $592 \mathrm{p}$.

2 Pour plus de détails, consulter notamment: Paul John MoRAN, op. cit., (chapitre premier), et Michel Morisset, op. cit., (cette thèse est en partie reprise dans L'agriculture familiale au Québec, Paris, Éditions l'Harmattan, 1987, 206 p.) ou encore, Jean-Pierre KESTEMAN et al., Histoire du syndicalisme agricole au Québec - UCC-UPA - 1924-1984, Montréal, Boréal Express, 1984, 327 p.

3 Cette estimation conservatrice est fondée sur des calculs effectués à partir de données du Recensement agricole de 1986 et de compilations spéciales tirées de l'Enquête sur la population active de Statistique Canada pour cette même année. Voir Paul John Moras, op. cit., (chapitre III.1). Selon les statistiques pertinentes les plus récentes, il y a eu en moyenne 37300 personnes salariées et de divers statuts en agriculture au Québec durant l'été 1988. Voir Statistique Canada, La population active, no de cat. 71-001, Ottawa, ministère des Approvisionnements et Services Canada, tableau 19, numéros de juin, juillet et août 1988 . 
4 Cf. SeRvice de MAIN-D'OeUVRE AGRICOLE DE L'UPA (SMA-UPA), Document de travail et recommandations du comité sur les problèmes de pénurie de main-d'oeuvre agricole, 3 juin 1988, p. 6. Voir aussi Paul John MoraN, op. cit., (chapitre III.2).

5 Cependant, les employeurs et les centres d'emploi concernés semblent noter chez les adolescents un faible attrait pour ce genre de travail. Ils ont donc peu d'espoir d'accroître ce type de main-d'oeuvre. Cf. SMA-UPA, op. cit., p. 15.

6 On évalue actuellement leur nombre à environ 1000 travailleurs. Sources: «Les journaliers agricoles», un reportage de Luc SIMARD, Le Magazine économique, 15 juillet 1989, Radio-Canada AM et «Le Québec horticole a grandement besoin de main-d'oeuvre mais les Québécois boudent le travail des champs», un reportage de Ginette LAMARCHE, Émission Présent-dimanche, 6 août 1989, Radio-Canada AM.

7 Au nombre d'environ 35 au début de la dernière décennie, les travailleurs saisonniers en provenance du Mexique ont été estimés à 725 personnes durant l'été 1989. Sources: GouvERNEMENT DU QUEBEC, Rapport sur le travail saisonnier dans le secteur agricole du Québec, par Michel Cournoyer, Québec, ministère de la Main-d'oeuvre et de la Sécurité du revenu, Services des politiques de main-d'oeuvre, Direction générale de la planification, avril 1987, pp. 3 et 4, et CEIC-MAPAQ-MMSR-UPA, Procès-verbal du Comité de concertation sur la main-d'oeuvre agricole, réunion du 29 septembre 1989 , p. 4.

8 Voir infra, note 49 et le texte correspondant.

9 Les chiffres présentés dans ce paragraphe sont fondés sur des données provenant du Recensement agricole de 1986. Voir Paul John MoRAN, op. cit., (chapitre III.3 et Tableau 14).

10 Ces chiffres sont tirés de quelques reportages écrits ou radiophoniques récents. Voir supra, note 6 et Paul John Moran, op. cit., p. 139, note 40.

11 Un examen spécifique des conventions collectives en vigueur en 1986 établissait à 175 le nombre de travailleurs agricoles syndiqués et ce, dans neuf établissements. Voir Paul John MORAN, op. cit., pp. 139 à 144.

12 Vraisemblablement, ces estimés de salaires bruts comprennent, dans le cas des ouvriers logés et nourris, une somme de $100 \$$ à titre de frais de pension assumés par l'employeur. Voir UPA, Service de la main-d'oeuvre de l'UPA - Rapport des activités 1987-1988, novembre 1988, tableau intitulé Salaires hebdomadaires bruts, note explicative, [s.p.].

13 Cf. SMA-UPA, op. cit., page 2, Tableau I.

14 Cf. ibid., et Paul John Moran, op. cit., p. 151 et 332, Annexe 2, Contrat-type de travail privilégié par le SMA-UPA et la Fédération des producteurs de lait du Québec, art. 8 et 9 .

15 Cf. Victor LARIVIÈRE, «En sept ans, 99 morts sur les fermes du Québec», La Terre de Chez Nous, édition du 7 juillet 1988 , p. 5.

16 Cf. UPA, "Ce que l'UPA attend des intervenants en matière de prévention», La prévention des accidents du travail en agriculture - Compte-rendu des journées d'étude des 19 et 20 décembre 1984, La Pocatière, Institut de technologie agricole, 1985, p. 101.

17 Pour une analyse du contrat individuel de travail, consulter: Claude D'Aoust, Le contrat individuel de travail en droit québécois, Montréal, La librairie de l'Université de Montréal, 1970, 108 p.; André RousSEAu, «Le contrat individuel de travail», Noël Mallette, dir., La gestion des relations de travail au Québec - Le cadre juridique et institutionnel, Montréal, McGraw-Hill, 1980, 642 p., p. 13 a 33; Robert P. GAGNon, Louis LeBel et Pierre Verge, Droit du travail, Québec, P.U.L., 1987, 933 p., p. 41 à 215; A. Edward AuST, Le contrat d'emploi, Cowansville, Les Editions Yvon Blais Inc., 1988, 235 p.; Georges AudET et Robert Bonноммe, Le congédiement en droit québécois en matière de contrat individuel de travail, $2^{\mathrm{e}}$ éd., Cowansville, Les Éditions Yvon Blais Inc., 1988, 487 p.

18 Il semble bien qu'aujourd'hui, cette disposition du Code civil n'ait plus d'utilité puisque, dans la mesure où la preuve testimoniale est admissible, une partie peut toujours utiliser son témoignage assermenté en sa faveur. Cf. Pierre-Basile Mignault, Le droit civil canadien, tome septième, Montréal, Wilson et Lafleur, 1906, 439 p., p. 378. 
19 Selon la catégorie à laquelle il appartient, le salarié québécois est soumis à un délai de prescription d'un an, de deux ans ou de cinq ans pour réclamer le salaire impayé. Voir Code civil, art. 2260(6), 2261(3) et 2262(3). Consulter A. Edward Aust, op. cit., p. 156 à 158; Robert P. GAGNON et al., op. cit., p. 214.

20 L.R.Q., c. N-1.1 (ci-après désignée sous l'abréviation LNT). De façon générale au sujet de cette loi, consulter notamment: Jean-Louis DubE et Nicola DiIorIo, Les normes du travail, Sherbrooke, Les Éditions Revue de Droit - Université de Sherbrooke, 1987, 442 p.; Gérard Hébert et Gilles TrUdeAU, Les normes minimales du travail au Canada et au Québec - Étude juridique et institutionnelle, Cowansville, Les Éditions Yvon Blais Inc., 1987, 192 p.

21 Cette loi s'applique à tous les salariés et à certains entrepreneurs dépendants tels que définis à son article 1 (10). Voir aussi l'article 2 de la loi.

22 Voir LNT, art. 93 et 94.

23 S.Q. 1940 , c. 39 ou L.R.Q., c. S-1.

24 L.R.Q., c. S-1, art. 1(a) et 2(a). Ces dispositions générèrent une jurisprudence qui définissait l'exploitation dans un sens large: Commission du salaire minimum (C.S.M.) c. Lucien Fleurant, Cour de magistrat d'Arthabaska, 6770, 28 octobre 1942, j. Marchand, (lin travaillé avec les machines placées dans la grande d'un agriculteur et achetées avec deux autres producteurs agricoles); C.S.M. c. Ferme Renil Ltée, [1968] B.R. 423, (élevage de volailles à une grande échelle), (à la page 424 de cette décision, on lit: «Agriculture means farming in its broadest sense and this includes what is commonly know as chicken farms.»); C.S.M. c. Chenil Laval Inc., Cour provinciale, district de Montréal, 500-02-012394-78, 30 octobre 1979, j. Turmey, (élevage de chiens).

Par contre, les activités commerciales proches de l'exploitation agricole sont assujetties à la loi: C.S.M. c. Gérard Lacoste et Herbert J. Irwin, Cour supérieure, district de Bedford, 12088, 7 février 1964, j. Cliche, (commerce d'animaux constituant l'activité la plus importante de la ferme où il était sis); C.S.M. c. Ernest Lier ou Camp-École d'équitation Lemoyne-D'Iberville, Cour provinciale, district de Québec, 79-526, 6 décembre 1972, j. Lemay, (camp-école d'équitation); C.S.M. c. Jardin du Pont de Québec Inc., Cour provinciale, district de Québec, 55-682, 18 juin 1973, j. Dussault, (entreprise de terrassement, de plantation d'arbres et de pose de tourbe); C.S.M. c. Dr. Guy Nadeau ou Ranch G. N., Cour provinciale, district de Québec, 87-308, 8 novembre 1973, j. Dussault, (pension et location de chevaux).

25 En effet, dans son mémoire présenté à la Commission parlementaire chargée d'étudier le projet de loi sur les normes du travail, l'Union des producteurs agricoles (UPA) demandait, à toutes fins pratiques, de reconduire l'exclusion contenue dans la Loi du salaire minimum. Voir UPA, Mémoire présenté à la Commission parlementaire du Travail et de la Main-d'oeuvre sur le projet de loi sur les normes du travail, mars 1979, 6 p. et annexe, document reproduit en partie dans le Journal des débats de l'Assemblée législative du Québec, 1979, Vol. 21 numéros 21 à 45, p. B-1069 à B-1077.

26 Voir Commission des normes du travail c. Lemoine Tropica Canada Inc., Cour provinciale, district de Montréal, 500-02-055568-823, 19 août 1986, j. Marleau, 6 p., (grossiste en plantes).

27 Cf. Jean-Louis DuBÉ et Nicola DiIorio, op. cit., p. 35-36. Ces auteurs renvoient à une décision qui incluait dans le terme «exploitation d'une ferme» de la Loi du salaire minimum un kiosque de vente rattaché à une ferme et essentiel à l'écoulement de sa production. Voir C.S.M. c. Fruits et légumes Hervey Roy Inc., Cour provinciale, district de St-François de Sherbrooke, 450-02-000546-80, 12 août 1981, j. Blanchette, 7 p., (kiosque de vente de produits agricoles).

28 Voir par analogie Commission des normes du travail c. Pépinière Fleur de Lys Inc., [1989] R.J.Q. 2249 (Cour du Québec), où le juge Monique Sylvestre propose la définition suivante d'agriculture: «l'agriculture comprend un aspect de transformation du milieu naturel en vue d'une production. Cette transformation exige une intervention humaine dont le degré 
variera selon le type d'agriculture.» (à la page 2250). Ce juge s'est inspiré d'une définition du mot agriculture retrouvée dans un dictionnaire usuel et qui inclut dans ce terme tant la production de végétaux que des animaux. Bien que la définition du juge Sylvestre ait été énonçée eu égard à l'article 54(7) de la Loi sur les normes du travail, elle permet d'entrevoir l'éventail de sens qu'il est possible de donner à l'expression «exploitation d'une ferme», formulée à l'article 3 de cette loi. La Commission des normes interprète aussi l'expression «exploitation d'une ferme» comme incluant l'entreprise qui élève des animaux de ferme. Voir: Commission DEs NORMES DU TRAVAIL, Loi sur les normes du travail - Règlement sur les normes du travail Loi sur la fête nationale - Interprétation et jurisprudence, $2^{\mathrm{e}}$ éd., Québec, Direction des affaires juridiques, Commission des normes du travail, 1988, 142 p., p. 12.

29 Cf. Commission des normes du travail (CNT) c. Maurice A. Hénault, Cour provinciale, district de Joliette, 705-02-001625-839, 25 mars 1985, j. Robillard, p. 11, (ferme de tabac exploitée avec moins de trois salariés mais qui doit recourir à des moments précis, chaque année, à une main-d'oeuvre supplémentaire). Voir aussi, CNT c. La ferme Drummond Inc., Cour provinciale, district de Drummond, 405-02-000724-82, 26 avril 1983, j. Biron, 8 p., (ferme ayant eu plus de trois salariès pendant 102 des 156 semaines considérées, application de la loi dans les circonstances). Pour sa part, la Commission des normes du travail suggère d'utiliser une période minimale d'une année pour déterminer la présence habituelle de plus de trois salariés dans une même ferme et son assujettissement à la loi. Voir COMMISSION DES NORMES DU TRAVAIL, op. cit., p. 12.

30 Cf. Jean-Louis Dube et Nicola Dilorio, op. cit., p. 36.

31 Cf. COMMISSION DES NORMES dU TRAVAIL, op. cit., p. 12.

32 LNT, art. 54(7). Pour conclure à l'application de cette exception, il faut d'abord vérifier si l'employeur exerce une activité agricole et, dans le cas d'une corporation, si elle constitue son activité principale. Dans l'affirmative, il faut ensuite s'assurer que le salarié effectue réellement des tâches propres au travailleur agricole. Voir notamment, $C N T$ c. Pépinière Fleur de Lys Inc., précitée à la note 28, p. 2250 et CNT c. Lemoine Tropica Canada Inc., précitée à la note 26, p. 5.

33 LNT, art. 54(5).

34 Voir LNT, art. 77(6).

35 R.R.Q. 1981, c. N-1.1, r. 3.

36 Id., art 2 (5) et (6).

37 Voir LNT, art. 88, ler et 2e alinéa.

38 Voir supra, note 8 et le texte correspondant.

39 Au moment d'écrire ces lignes, le ministre de la Main-d'oeuvre, de la Sécurité du revenu et de la Formation professionnelle a déjà déposé un avant-projet de loi intitulé Loi modifiant la loi sur les normes du travail et d'autres dispositions législatives (Éditeur du Québec, juin 1989). Cet avant-projet de loi propose de retirer l'exclusion générale, formulée à l'article 3 de la loi actuelle, et de ne l'appliquer qu'aux normes portant sur le salaire minimum, le versement du salaire et le bulletin de paie, les retenues salariales, les frais d'hébergement, la semaine normale de travail ou le temps supplémentaire. Par ailleurs, aucune des autres exclusions spécifiques à l'égard des travailleurs agricoles n'est modifiée par cet avant-projet de loi.

40 L.R.Q., c. F-1.1.

41 Voir notamment l'article 7 de cette loi. Cette disposition exige de tout travailleur au moins dix jours de salaire ou d'indemnité tenant lieu de salaire, entre le ler et le 23 juin, pour pouvoir bénéficier du congé compensatoire ou de son équivalent pécuniaire.

42 L.R.Q., c. S-2.1. Au sujet de cette loi, voir notamment: Denis BRADET, Bernard Cliche, Martin Racine et France Thibault, Droit de la santé et de la sécurité du travail - La loi et la jurisprudence commentée, Cowansville, Les Éditions Yvon Blais Inc., 1986, 300 p.

43 Voir supra, notes 15 et 16 et le texte les accompagnant. 
44 Actuellement, seuls deux des six grands secteurs d'activité économique sont assujettis au règlement portant sur les comités de santé et sécurité et à celui relatif au représentant à la prévention. Voir: Règlement sur les comités de santé et de sécurité au travail, (1983) 115 G. O. II, 4209 et Règlement sur le représentant à la prévention dans un établissement, (1984) 116 G. $O$. II, 4195. Un troisième secteur a été assujetti au règlement en matière de programme de prévention. Voir: Règlement sur le programme de prévention, (1982) 114 G. O. II, 2373 modifié par le décret 747-83, (1983) 115 G. O. II, 1927 et par le décret 361-85, (1985) 117 G. O. II, 1552. L'industrie agricole fait partie du sixième et dernier secteur auquel s'appliquera possiblement cette réglementation.

45 Loi sur les accidents du travail et les maladies professionnelles, L.Q. 1985, c. 6 ou L.R.Q., c. A-3.001.

46 S.Q. 1930-1931, c. 100 ou L.R.Q., c. A-3. Pour une analyse historique du régime québécois à cet égard, consulter notamment: Katherine LIPPEL, «Droit des travailleurs québécois en matière de santé - 1885-1981», (1981-82) 16 Revue juridique Thémis 329.

47 Voir Loi modifiant la Loi des accidents du travail et d'autres dispositions législatives, L.Q. 1978 , c. 57 , art. 67 et 94.

48 Voir Proclamation du 19 novembre 1980, (1980) 112 G. O. II, 10 décembre 1980, p. 6661 ou le Décret 3624-80. Voir aussi: CoMmission DE LA SANTÉ ET DE LA SECCURITÉ DU QuÉBEC, Rapport annuel 1980, Québec, Direction des communications, CSST, 1981, p. 37.

49 L.R.Q., c. C-27.

50 L'ancêtre du Code du travail, la Loi des relations ouvrières (S.Q. 1944, c. 30 ou S.R.Q. 1941, c. 162A) excluait «les personnes employées dans une exploitation agricole», laquelle signifiait «une ferme mise en valeur par l'exploitant lui-même ou par l'entremise d'employés» (art. 2, alinéa a, 4e paragraphe, et alinéa b). Cette exclusion générale disparut lors de l'adoption du Code du travail en 1964.

51 La première version de cette disposition, alors l'article 20(5) du Code du travail de 1964 (S.Q. 1964, c. 45), se lisait comme suit:

Lorsque moins de trois personnes sont ordinairement employées à l'exploitation d'une ferme, elles ne sont pas réputées des salariés pour les fins de la présente section. (L'italique est de nous)

Cet alinéa fut modifé en 1965 (S.Q. 1965, c. 50, art. 2), pour se lire ainsi:

Les personnes employées à l'exploitation d'une ferme ne sont pas réputées être des salariés pour les fins de la présente section à moins qu'elles n'y soient ordinairement et continuellement employées au nombre de trois au moins. (L'italique est de nous)

La disposition prit sa forme actuelle lors des amendements apportés au Code en 1969 (S.Q. 1969, c. 49, art. 9).

52 Coopérative des pomiculteurs du Québec c. Syndicat de la Coopérative des pomiculteurs du Québec et al., [1971] T.T. 254, p. 255 et 256. La décision du commissaire-enquêteur est reproduite à (1971) C.E. 663.

53 Les patates québécoises Ltée c. Union des employés de commerce, Local 503 (CTCFTQ), Tribunal du travail, district de Québec, 200-28-00081-809, 12 décembre 1980, j. Brière, p. 4.

54 Corp. de l'École d'agriculture de Ste-Croix c. Syndicat des émployés de l'École d'agriculture de Ste-Croix, [1984] T.T. 232, p. 233 et 234.

55 Ibid., p. 234.

56 Voir supra, note 11 et le texte correspondant.

57 L.R.Q., c. D-2 (ci-après citée sous l'abréviation LDCC). Sur cette loi, consulter notamment: Jean-Louis DuBE, Décrets et comités paritaires - L'extension juridique des conventions collectives, Sherbrooke, Les Éditions Revue de Droit, Université de Sherbrooke, $1990,376 \mathrm{p}$. 
58 LDCC, art. 1(a) et 29(a).

59 Cf. Robert P. GAGNON et al., op. cit., p. 478.

60 Partie I de la Loi constitutionnelle de 1982, adoptée comme Annexe B de la Loi de 1982 sur le Canada, R.-U., 1982, c. 11.

61 La compétence d'un tribunal administratif de déclarer contraire à la Charte la loi qu'il applique demeure une question controversée. Par exemple, la Cour d'appel de l'Ontario a reconnu cette compétence à l'Ontario Labour Relations Board dans l'affaire Cuddy Chicks Limited c. Ontario Labour Relations Board et al., (1990) 44 C.R.R. 75n (O.C.A.), requête pour permission d'en appeler acceptée par la Cour suprême du Canada le 22 février 1990. À l'inverse, la Cour fédérale d'appel a refusé une telle compétence à un organisme administratif exerçant des pouvoirs d'adjudication. Voir: Attorney General of Canada c. Vincer, (1989) N. R. 352 et Procureur général du Canada c. Sirois, (1989) 90 N. R. 39. De façon plus générale sur cette question, consulter parmi d'autres: Gilles PEPIN, «La compétence des cours inférieures et des tribunaux administratifs de stériliser, pour cause d'invalidité ou d'ineffectivité, les textes législatifs ou réglementaires qu'ils ont pour mission d'appliquer», (1987) 47 La Revue du Barreau 509.

62 Charte canadienne des droits et libertés, art. 1.

63 On pourrait croire que l'exclusion partielle des ouvriers agricoles du Code du travail (voir supra, note 51 et le texte l'accompagnant) restreint aussi la liberté d'association qui est protégée par l'article 2 de la Charte. La Cour suprême du Canada a toutefois refusé d'inclure dans la liberté d'association le droit à la négociation collective et le droit de grève. Voir à cet effet les trois décisions suivantes: Re Public Service Employee Relations Act, [1987] 1 R.C.S. 313; P.S.A.C. c. Canada, [1987] 1 R.C.S. 424; R.W.D.S.U. c. Saskatchewan, [1987] 1 R.C.S. 460.

64 R.S.O. 1980, c. 228. L'exclusion est énoncée à l'article 2(b) de cette loi.

65 La Cour d'appel de l'Ontario a récemment reconnu la compétence du Labour Relations Board pour examiner une telle contestation. Cette décision a été rendue dans l'affaire Cuddy Chicks Limited, précitée à la note 61 .

66 [1989] 1 R.C.S. 143. Dans cette affaire, on contestait l'exigence de la citoyenneté canadienne imposée par la Law Society à tout candidat à la pratique du droit en ColombieBritannique.

67 Ibid., p. 174.

68 Le juge McIntyre écrit même la phrase suivante: «[1]es motifs énumérés eux-mêmes et les autres motifs possibles de discrimination reconnus au par. 15(1) doivent, dans les deux cas, recevoir une interprétation large et libérale». Ibid., p. 175.

69 C'est du moins l'opinion de l'auteur Dale Gibson qui a récemment publié une étude détaillée sur la question. Voir: Dale GiBson, The Law of the Charter: Equality Rights, Toronto, Carswell, 1990, 371 p., p. 143 et ss.

70 [1986] 1 R.C.S. 103. Pour une étude du test proposé par cette décision en relation avec l'article 15(1) de la Charte, consulter: Dale Gibson, op. cit., p. 261 et ss.

71 Ibid., p. 138.

72 Ibid., p. 139.

73 Idem.

74 L'auteur Gibson est cependant prudent à ce sujet. Il se fonde en cela sur l'opinion laconique mais unanime de la Cour suprême du Canada exprimée dans l'affaire Re Workers ' Compensation Act, 1983 (T.N.), [1989] 2 R.C.S. 922. Dans ce dossier, on contestait, sur la base de l'article 15(1) de la Charte, le fait que l'indemnisation accordée aux travailleurs visés en vertu de la loi sur les accidents du travail de Terre-Neuve tienne lieu et place de tous les droits et actions auxquels un travailleur pourrait autrement recourir. La Cour écrit ce qui suit: «La situation des travailleurs et des personnes à charge n'est aucunement analogue aux situations énumérées au par. 15(1), exigence posée par la majorité dans l'affaire Andrews pour permettre 
le recours au par. 15(1).» (à la page 924, voir aussi Dale GIBSON, op. cit., p. 255-257). Le laconisme de la Cour suprême s'explique peut-être par la longue décision de la Cour d'appel de Terre-Neuve qu'elle confirme. Dans sa décision, la Cour d'appel concluait que le régime de compensation des victimes de lésions professionnelles n'est pas discriminatoire au sens de l'article 15(1) de la Charte parce qu'il ne crée pas une distinction injuste ou déraisonnable à l'égard de ces citoyens. Il en est ainsi parce que ce régime, dans son ensemble, bénéficie aux travailleurs qui y sont assujettis. La Cour d'appel ne discute cependant pas des motifs de discrimination qui seraient prohibés par l'article 15(1). Voir: Re Sections 32 and 34 of the Workers' Compensation Act (Nfld.) (1988) 67 Nfld. and P.E.I. Reports 16. Par ailleurs, tout en abordant pas la question de la nature des motifs de discrimination, la Cour fédérale d'appel souligne, dans une décision récente, que traiter l'industrie du débardage différemment des autres industries, en matière de relations de travail, ne viole pas le droit à l'égalité de l'article 15(1) de la Charte. Voir: Terminaux portuaires ciu Québec inc. c. Association des employeurs maritimes, (1989) 89 N. R. 278; requête pour permission d'en appeler rejetée par la Cour suprême du Canada, (1989) 92 N. R. 240.

75 À ce sujet, consulter notamment: John BANK, «Farm Workers: Victims outside the Law», (1975) The Labour Gazette 368 ou David M. BEATTY, Putting the Charter to Work Designing a Constitutional Labour Code, Montréal, McGill-Queen's University Press, 1987, 252 p., p. 89 et ss. Par ailleurs, notons que l'UPA s'est encore récemment opposée à toute modification des normes du travail à l'égard du secteur agricole. Cf. UNION DES PRODUCTEURS AGRICOLES, «Lettre du président général, Jacques Proulx, au ministre André Bourbeau concernant l'avant-projet de loi sur les normes du travail", datée du 29 janvier 1990,8 p., p. 1 et 6 . Voir également supra, notes 25 et 39.

76 Voir notamment à cet égard: Kathryn Neilson et Innis Christie, «The Agricultural Labourer in Canada: A Legal Point of View», (1975) 2 Dalhousie Law Journal 330, p. 359 et ss.

77 L.R.Q., c. C-12. La Charte québécoise lie la Couronne: on peut donc l'invoquer pour contester le contenu d'une loi adoptée par l'Assemblée nationale (art. 54). Le législateur peut cependant déroger aux dispositions de la Charte en l'indiquant expressément dans la loi en question (art. 52). Aucune loi québécoise du travail, en vigueur et d'application générale, ne contient une telle clause dérogatoire.

78 La Charte québécoise établit que la liberté d'association constitue une liberté fondamentale. Cependant, compte tenu de l'interprétation de la notion de liberté d'association faite par la Cour suprême dans le cadre de l'article 2 de la Charte canadienne (voir supra, note 63), nous croyons peu utile de fonder sur cette liberté une contestation de la disposition du Code $d u$ travail qui concerne les ouvriers agricoles.

79 De façon générale, voir à ce sujet: Madeleine $\mathrm{C}_{\mathrm{ARON}}$, «Le droit à l'égalité dans le Code civil et dans la Charte québécoise des droits et libertés", (1985) 45 La Revue du Barreau 345 , p. 356 et ss.; Pierre Carignan, «L'égalité dans le droit: une méthode d'approche appliquée à l'article 10 de la Charte des droits et libertés de la personne», (1987) 21 Revue juridique Thémis 493, p. 526 et ss. Par ailleurs, le deuxième alinéa du Préambule de la Charte québécoise se lit: «Considérant que tous les êtres humains sont égaux en valeur et en dignité et ont droit à une égale protection de la loi." (L'italique est de nous) On ne peut cependant s'autoriser de cette disposition pour soutenir que la Charte reconnaît un droit autonome à l'égalité. En effet, le dispositif d'une loi (par exemple, l'article 10 de la Charte) doit avoir préséance sur son préambule. En la matière, consulter: Pierre-André Côté, Interprétation des lois, Cowansville, Les Éditions Yvon Blais Inc., 1982, 695 p., p. 41 à 43.

80 Voir à ce sujet: Johnson c. Commission des affaires sociales, [1984] C.A. 61. Dans cette affaire, La Cour d'appel examinait la validité de l'article 8 de la Loi de l'aide sociale qui refuse aux grévistes le droit aux prestations d'aide sociale. 
81 Johnson c. Commission des affaires sociales, précitée, p. 69 et 70 . La Cour reprend ici une définition utilisée par le juge Tôth dans l'affaire Commission des droits de la personne $\mathrm{c}$. Centre hospitalier St-Vincent-de-Paul de Sherbrooke, Cour supérieure, district de St-François, 450-05-000856-78, le 7 septembre 1978.

82 À cet égard, voir entre autres: André COLLARD, «La condition sociale: est-ce vraiment un motif de discrimination?", (1987) 47 La Revue du Barreau 188, p. 188.

83 Comparer cette définition avec celle utilisée par la Cour d'appel dans l'affaire Johnson. Voir supra, note 81.

84 Voir supra, note 3.

85 Le Code du travail n'exclut pas totalement les travailleurs agricoles de son champ d'application. Il leur retire le bénéfice de l'accréditation syndicale. Cependant, les avantages les plus significatifs du Code ne sont donc pas disponibles à cette catégorie de salariés.

86 Voir supra, note 8 et le texte correspondant.

\section{Agricultural Wage Earning in Québec}

The subject of this article is centered upon non-family farmworker in the Québec agrarian sector. In the first instance, it describes the principal socioéconomic characteristics of these workers. Afterwards, it analyses to what degree farm-workers are subject to Québec labour laws. Finally, it discusses the validity of various legal exceptions and exclusions aimed at agricultural employees in light of the Canadian and Québec charters of rights and freedoms.

There are at least 15000 non-family farmworkers in Québec. Approximately fifty percent of these employees are permanent workers, hired for the most part on livestock and poultry farms. Most of the non-family farmworkers are young male adults and of French-canadian origin. Some women and foreign farmworkers are to be found, but mostly on vegetable or fruit farms, and on a seasonal basis.

Only a few agricultural employees are unionized. Farmworkers earn low wages for long workweeks. Agricultural work also provides for only a few legal holidays, and is characterized by a high level of occupational accidents.

To what extent does Québec labour laws apply to employees in the agricultural sector? This question calls for a not so clear-cut answer.

The rules set forth in the Civil code pertaining to individual labour contracts are entirely applicable to the agricultural sector. So much can be said for the rules established in the Act Respecting Occupational Health and Safety and in the Act Respecting Industrial Accidents and Occupational Diseases. These two laws do not create any exclusions or exceptions whatsoever with regard to farmworkers.

On the other hand, these employees are deprived, in part or in whole, from the benefits of other important legislations concerning work conditions and industrial relations. Thus, only agricultural employers with at least four permanent workers are 
subject to the Act Respecting Labour Standard. Furthermore, employees who fall within the application of this law may not benefit from all the minimal standards that it provides.

The Labour Code, in its application to farmworkers, is in no way different. Effectively, persons employed on a farm which has fewer than three full time workers are not considered «employees» as determined in the Labour Code, and are thus not entitled to union representation or certification. Considering the numerous farms in Québec that hire fewer than three permanent workers, the great majority of farmworkers are deprived of the advantages brought about by trade-unions through collective bargaining. Finally, the Act Respecting Collective Agreement Decrees does not cover «agricultural exploitations» whatever the number of farmworkers in their employ.

Farmworkers are unfavourably treated by our labour laws when compared to employees working in other sectors of our economy. In so being, they are victims of discrimination based upon their occupational activity. This discrimination could be judicially challenged on the basis of section 15 of the Canadian Charter of Rights and Freedoms. Section 15 recognizes and guarantees the equality of all before and under the law. In spite of an uncertain jurisprudence on this subject, the door remains open for a court challenge. Futhermore, this occupational discrimination could also be attacked on the basis of some provisions of the Québec Charter of Human Rights and Freedoms which prohibits any form of discrimination based upon «social condition». Here again, farmworkers will have to overcome a jurisprudence which has favoured, up to now, a restrictive definition of «social condition».

\section{ACQUISITION OU FUSION D'ENTREPRISES ET EMPLOIS}

Préface, Gilles LAFLAMME - Introduction, Laurent BÉLANGER, Carla LIPSIG, Fernand MORIN, Michel PERUSSE - Acquisition ou fusion d'entreprises et emplois: la problématique sous-jacente, Fernand MORIN - Rappel de quelques expériences vécues, Mare BELANGER, Lola LE BRASSEUR, Paul L'TTALIEN, Marius MENARD - Aspects et implications juridiques des restructurations, André C. CÓTE, Claude FONTAINE, Paul LESAGE - Le droit et le dewoir à linformation, Georges ANGERS, Normand GAGNON, Jean SIMARD - Les conditions de travail au lendemain d'une fusion ou d'une réorganisation, André LAMARCHE, Michel GAUTHIER, Michel BLAIS, Denise GAGNON - Fusion d'entreprises publiques, Astrid GAGNON, Sylvain BLANCHETTE, Pierre QUINTAL - Les acteurs d'une fusion ou d'une prise de pouvoir, Alain GOSSELIN, Jean-Guy FRENETTE, Denis DIONNE - Le libre-échange canado-américain et le marché du travail, Clément GODBOUT, Claude RIOUX - Annexe: Acquisitions, fusions, offres publiques d'achat: notions de base et facteurs considérés, Léontine ROUSSEAU.

ISBN 2-7637-7205-6

1 volume, 1989, 264 pages, $26 \$$

LES PRESSES DE LUNTVERSITELAVAL

AV. DE LA MÉDECINE, CITE UNIVERSITARE, STE-FOY G1K 7P4 Tél.: (418) 656-5106 Télécopieur: (418) 656-3476 